\title{
Análisis de los cambios en las propiedades mecánicas de materiales de subrasante por la adición de materiales poliméricos reciclados
}

Analysis of the changes in the mechanical properties of subrasant materials by the addition of recycled polymeric materials

Análise das mudanças nas propriedades mecânicas de materiais de subleito por adição de materiais poliméricos reciclados

\section{Erika Julieth Serrano Rodríguez ${ }^{1}$ Edgar Alexander Padilla González²}

Recibido: septiembre 15 del 2018

Aprobado: noviembre 30 del 2018

Disponible en línea: enero 15 del 2019

Cómo citar este artículo:

E. Serrano Rodríguez y E. Padilla González, "Análisis de los cambios en las propiedades mecánicas de materiales de subrasante por la adición de materiales poliméricos reciclados", Revista Ingeniería Solidaria, vol. 25, n. ${ }^{\circ}$ 1, 2019. DOI: https://doi.org/10.16925/2357-6014.2019.01.

Artículo de investigación. https://doi.org/10.16925/2357-6014.2019.01.01

Universidad de la Salle, Bogotá, Colombia

ORCID: https://orcid.org/0000-0001-6989-133X

Correo electrónico: serika30@unisalle.edu.co

2 Universidad de la Salle, Bogotá, Colombia

ORCID: https://orcid.org/0000-0003-0730-2776 


\section{Resumen}

Introducción: este artículo es producto de la investigación "Análisis de la resistencia y durabilidad de un suelo de cemento adicionando material no biodegradable polietileno malla Raschel (polisombra) reciclada en diferentes porcentajes en relación con el peso del suelo", desarrollada en la Universidad de la Salle durante el 2016.

Problema: identificación de las variaciones en las propiedades mecánicas de materiales de subrasante por la adición de materiales poliméricos.

Objetivo: recopilar las principales investigaciones sobre las modificaciones de las propiedades de subrasante por medio de adición de fibras naturales y sintéticas, con el fin de tener una base teórica que justifique la aplicación de estas adiciones a nivel constructivo.

Metodología: recopilación de bibliografía relevante sobre modificación de subrasante con polímeros y la evaluación del cambio en sus propiedades mecánicas, síntesis y discusión de resultados.

Resultado: el $65 \%$ de las investigaciones encontradas corresponde a estudios sobre las modificaciones de la resistencia a la compresión; el 30 \% de ellas están dirigidas a estudios enfocados a estudiar la respuesta de los esfuerzos de tracción cuando son fibras poliméricas; y el otro $5 \%$ evalúa propiedades como la fatiga o resistencia a la tensión axial.

Conclusiones: se apunta un aumento considerable en la resistencia de compresión, que varía según el tipo o material a mezclar con el suelo y cemento.

Originalidad: este artículo presenta la sustitución de materias primas de la subrasante con fibras naturales o sintéticas recicladas.

Limitaciones: hasta ahora son pocas las investigaciones que se han encaminado a desarrollar modificaciones de los materiales en la subrasante con fibras naturales; por consiguiente, la información para analizar se ve limitada.

Palabras clave: subrasante, suelo-cemento, fibras naturales, fibras sintéticas.

\section{Abstract}

Introduction: This review is a compilation of several researches. However, it is based on a thesis called "Análisis de la resistencia y durabilidad de un suelo cemento adicionando material no biodegradable polietileno malla Raschel (polisombra) reciclada en diferentes porcentajes en relación con el peso del suelo", that was carried out at Universidad de La Salle in 2016.

Problem: The identification of the variations in the mechanical properties of materials of subgrade by the addition of polymeric materials.

Objective: to compile the main research on the modifications of subgrade properties through the addition of natural and synthetic fibers, in order to have a theoretical basis that justifies the application of these additions at constructive level.

Methodology: Relevant references compilation referring to the modification of subgrade with polymers and the evaluation of the change in his mechanical properties, synthesis, and discussion of results.

Result: $65 \%$ of the investigations correspond to studies on changes in compressive strength, $30 \%$ of them are directed to focused studies to study the efforts of traction's answer when they are polymeric fibers, and the other $5 \%$ evaluates properties as the fatigue or resistance to the axial tension.

Conclusions: A considerable increase in the resistance of compression is denoted, which changes according to the type or material to mixing with the soil and cement.

Originality: This article presents the substitution of raw materials of the subgrade with recycled natural or synthetic fibers. 
Limitations: There are few researches made with natural fibers, so it is limited to the information found.

Keywords: Soil, cement floor, natural fibers, synthetic fibers.

\section{Resumo}

Introdução: este artigo é produto da pesquisa "Análise da resistência e durabilidade de um solo-cimento adicionado de material não biodegradável polietileno Raschel (tela de sombreamento) reciclado em diferentes porcentagens em relação com o peso do solo", desenvolvida na Universidad de la Salle durante 2016.

Problema: identificação das variações nas propriedades mecânicas de materiais de subleito por adição de materiais poliméricos.

Objetivo: recompilar as principais pesquisas sobre as modificações das propriedades de subleito por meio da adição de fibras naturais e sintéticas, com a finalidade de ter uma base teórica que justifique a aplicação dessas adições a nível construtivo.

Metodologia: recompilação de bibliografia relevante sobre modificação de subleito com polímeros e avaliação da mudança nas suas propriedades mecânicas, síntese e discussão de resultados.

Resultado: $65 \%$ das pesquisas encontradas correspondem a estudos sobre as modificações da resistência à compressão; $30 \%$ delas estão dirigidas a estudos enfocados em estudar a resposta dos esforços de tração quando são fibras poliméricas, e $5 \%$ avaliam propriedades como a fadiga ou a resistência à tensão axial.

Conclusões: aponta-se um aumento considerável na resistência de compressão, que varia de acordo com o tipo ou material a ser misturado com o solo e o cimento.

Originalidade: este artigo apresenta a substituição de matérias-primas do subleito com fibras naturais ou sintéticas recicladas.

Limitações: até agora são poucas as pesquisas que se dedicaram a desenvolver modificações dos materiais no subleito com fibras naturais, consequentemente, a informação disponível para análise é limitada.

Palavras-chave: subleito, solo-cimento, fibras naturais, fibras sintéticas.

\section{Introducción}

Según su composición, el suelo puede entenderse como un material trifásico: es decir, se compone de fase sólida, líquida y gaseosa [1] de tamaños de partículas variables y organización diferente, lo que explica la existencia de suelos de múltiples estructuras.

Uno de los materiales más utilizados en la construcción de subrasante es el suelo-cemento. Este es una mezcla homogénea entre el suelo, con unas características granulométricas definidas, y determinadas cantidades de cemento Portland y agua, que se compacta para obtener altas densidades y curado, de modo tal que se produzca un endurecimiento efectivo. El material obtenido se considera resistente a los esfuerzos de compresión, prácticamente impermeable, termoaislante y estable en el tiempo [1]. Este se ha vuelto importante en el desarrollo dentro de la ingeniería debido a las funciones geotécnicas del material, además de los beneficios ecológicos que trae al aumentar el rendimiento mecánico de los suelos, reduciendo a su vez los costos 
de infraestructura. Sin embargo, posee algunas limitaciones como poca resistencia mecánica, muros de gran espesor y vulnerabilidad a los agentes atmosféricos [2].

Existen diversos sistemas de estabilización de suelos de subrasante en el mundo, tales como la estabilización mecánica, que puede realizarse compactando el material o colocando un material de grano grueso y fino homogéneo sobre la subrasante; la estabilización con aditivos químicos, que alteran las propiedades fisicoquímicas del suelo a tratar mediante la generación de un incremento como cemento, limo y asfalto bituminoso; y la estabilización mediante geotextiles y geosintéticos [3]. Entre los procesos de estabilización más conocidos se encuentra la adición de cal al material de subrasante, en el que las variaciones en las propiedades del suelo incluyen reducción de la humedad natural, modificación de la granulometría, reducción del índice de plasticidad, reducción del potencial de cambio volumétrico y modificación de las características de compactación, entre otras [4].

Con lo dicho, el presente artículo comprende una recopilación de los principales hallazgos acerca de las modificaciones en las propiedades de la subrasante cuando se adicionan fibras naturales o sintéticas de polímeros, con el fin de generar una base teórica que justifique la implementación de estas adiciones a nivel constructivo y, de igual forma, muestre los elementos sobre los cuales se requiere más investigación. Así entonces, se muestran las características representativas principales de la subrasante y luego se describen los resultados obtenidos por los investigadores cuyos trabajos han ampliado el conocimiento en el área.

\section{Revisión de literatura}

López-Lara, Hernández-Zaragoza y Horta-Rangel explican que las arcillas expansivas sufren cambios de volumen debido a las variaciones en su humedad por infiltración [5]. Cualquier construcción que descanse sobre este tipo de suelos experimentará deformaciones causadas por la expansión de la arcilla. Dichos movimientos se traducen en grietas sobre muros y el levantamiento de pisos; por esto, se buscan soluciones para reducir los cambios volumétricos de la arcilla. En esta investigación se utilizó el poliuretano. Se concluye en dicho estudio que la mezcla suelo-polímero no mejora las propiedades de resistencia, aspecto que no es primordial en un suelo expansivo por presentar frecuentemente altas resistencias. Adicionalmente, uno de los resultados más significativos dentro de la investigación nombrada alude a la expansión: las pruebas mostraron que la mezcla suelo-polímero tiene una reducción en la expansión cercana al 40 \% frente a la expansión del suelo natural. 
Moreno-Navarro, Sol Sánchez y Rubio-Gámez examinaron el comportamiento estructural de las mezclas bituminosas utilizadas en las capas de la superficie de la carretera (teniendo en cuenta los costos y el rendimiento) [6], al tiempo que analizaron la viabilidad del uso de estos materiales para la rehabilitación de las vías de volumen de tráfico ligero-medio. Los resultados demostraron que los materiales modificados con polímeros podrían ser más eficientes desde el punto de vista estructural y económico que los no modificados, con lo que ofrecen una solución a los diseñadores para la rehabilitación de carreteras de volumen de tráfico ligero-medio.

Terreros-Caicedo, por su parte, afirma que los suelos expansivos formados con gran cantidad de minerales de arcilla se caracterizan por la absorción y retención de agua, lo cual ayuda a que el volumen del mineral aumente $y$, una vez se seque, disminuya (retracción) [7]. Este fenómeno es perjudicial para la construcción: puede afectar la cementación de tramos en tanto podría ocasionar hundimiento. Para dar solución a estos problemas, se experimentó estabilizarlo por medio de polímeros; ello alteró de manera significativa las condiciones físico-mecánicas del suelo y aumentó su elasticidad.

Respecto a la inclusión de geoceldas, en el ámbito internacional las investigaciones se han enfocado en estudios analíticos, numéricos y experimentales. De modo específico, los estudios analíticos desarrollados han estado dirigidos hacia la evaluación en los cambios sobre la capacidad de carga, el incremento de la presión de confinamiento y la falla a la tensión [8].

En el ámbito nacional, el trabajo de Duarte y Sierra [9] tuvo como fin evaluar los esfuerzos y deformaciones de un suelo de Bogotá que fue estabilizado con un sistema de geoceldas, por medio de la comparación entre los esfuerzos y asentamientos inducidos al aplicar una carga monotónica y ciclos de carga y descarga en una subrasante sin estabilizar, una estabilizada con un sistema de geoceldas y una mejorada con un sistema convencional. Los resultados obtenidos mostraron que utilizar un sistema de geoceldas y uno convencional reduce los esfuerzos y asentamientos en una subrasante; los asentamientos presentan una reducción del 34 \% al utilizar el primero en comparación con el segundo. Por otra parte, los esfuerzos se reducen en un 53 \% al utilizar un sistema de geoceldas como alternativa de estabilización respecto a un sistema convencional.

En la investigación realizada por Cuelho y Perkins se construyeron secciones de prueba para comparar el rendimiento operativo de geosintéticos en la estabilización de subrasantes [10]. Se edificaron 12 geosintéticos formados por una variedad de geomallas y geotextiles, además de secciones de prueba para evaluar el efecto que la resistencia de la subrasante, el grosor de la capa base y la presencia del geosintético 
tienen en el rendimiento. Por medio de una regresión lineal, en la que se usó "Iongitudinal rut" como indicador de rendimiento, se determinó que la rigidez es el factor que evalúa el efecto de la resistencia de la subrasante y el grosor de la capa base.

Múltiples secciones de prueba de control del estudio nombrado se construyeron para evaluar el efecto de la resistencia de la subrasante, el grosor de la capa base o la presencia del geosintético. Aunque los materiales geotextiles utilizados durante el estudio mostraron un buen rendimiento como estabilización de la subrasante, las propiedades del material asociadas con este último fueron difíciles de establecer debido al número limitado de secciones de prueba, así como a la falta de pruebas relevantes para caracterizar de forma adecuada este tipo de materiales en esta aplicación. Utilizando la rutina longitudinal como el principal indicador de rendimiento, se determinó, a través de un análisis de regresión lineal, que la rigidez de las uniones de geomalla en la dirección transversal a la máquina se correlacionaba mejor con el rendimiento en esta aplicación y bajo estas condiciones. A través de este conocimiento, la ecuación de diseño asociada con el método Giroud-Han fue calibrada para hacer que la rigidez de la unión de la geomalla en la dirección transversal a la máquina fuera la propiedad primaria del geosintético, reemplazándose así el módulo de estabilidad de apertura de la geomalla

Un estudio realizado por Geiman permitió comparar la mejora en la resistencia a la compresión de varios estabilizadores, entre los que se encontraba un polímero sintético líquido. Los resultados permitieron identificar que la mejora en las propiedades de las mezclas analizadas varía entre el 13 y el 20 \% [11].

En el estudio realizado por Khan se analizó el uso de polietileno de alta densidad (hdpe) proveniente de residuos, a fin de observar el comportamiento de la subrasante [12]. Las pruebas se realizaron en suelos donde las fibras habían sido utilizadas con diferentes longitudes proporciones (0 a 6 \%). Se constató que el uso de fibra ayuda a mejorar la resistencia del suelo; y el beneficio de refuerzo aumenta conforme crece el contenido de la fibra. El material se puede utilizar en cursos base para construir caminos rurales sobre arcilla saturada.

Li Chen y Deng-Fong Lin realizaron un estudio por medio del cual se analizó el comportamiento de la ceniza incinerada proveniente de lodos (issa) en una mezcla 4:1 como estabilizador para mejorar la resistencia del suelo blando, cohesivo y de subrasante [13]. Se realizaron pruebas del valor del pH, los límites de Atterberg, la compactación, el California Bearing Ratio (cbr), la resistencia a la compresión no confinada y la compresión triaxial. La resistencia a la compresión mejoró entre 3 y 7 veces; el hinchamiento se redujo entre 10 y $60 \%$; y los valores de cbr mejoraron hasta 30 veces frente a las muestras no tratadas. 
Hufenus et al. encontraron un aumento significativo en la capacidad de carga de una capa reforzada con geosintético cuando su espesor es inferior a 0,5 m, y en suelos con cbr inferior o igual a 2. De la misma manera, observaron que la compactibilidad de capas delgadas puede mejorar con el reforzamiento geosintético en suelos con un cbr inferior a 3 [14].

\section{Metodología}

Entre los diferentes estudios observados se encuentran puntos comunes en lo que atañe a los materiales y métodos implementados. A este respecto, Perugachi y Trajano realizaron varios ensayos para analizar cuál alternativa se podría utilizar en la estabilización de suelos subrasantes de tipo arena arcillosa. Al suelo natural se le añadieron porcentajes de toba volcánica, arena, cemento y cal, con el fin de determinar los cambios en su plasticidad y la capacidad portante del suelo [15]. Se determinó que el elemento que aportó la capacidad portante al suelo fue el cemento; en cambio, la cal y demás elementos no causaron ningún cambio en este. Por lo anterior, el suelo y el cemento es la mezcla por evaluar junto a los materiales reciclables para la adición a la subrasante.

\subsection{Suelos aptos para mezclas de suelo-cemento}

Se considera que un suelo es apto para componer una mezcla de suelo-cemento cuando este exige un consumo de cemento entre el 5 y el $12 \%$ en relación con el peso del suelo. De manera complementaria, estos suelos deben ser estables en la contracción y tener una absorción de agua idónea que, además, tenga una resistencia adecuada en un tiempo mínimo [1].

Existen diferentes criterios para evaluar la calidad del suelo-cemento [1]: los suelos que se usen para las mezclas de este último no pueden contener partículas que superen 7,5 cm de espesor, a la vez que el limite líquido y el índice plástico deben ser inferiores al $40 \%$ y al $18 \%$, respectivamente; de lo contrario, se afectarían su trabajabilidad y plasticidad. Seguidamente, se adicionarán tres diferentes porcentajes de pet (5\%, $10 \%$ y $15 \%$ ), que serán incluidos en peso y no en volumen. El material por estabilizar debe cumplir con los requisitos establecidos en el Artículo 341 de 2007 del Instituto Nacional de Vías (Invías) [16]. 


\subsection{Cemento Portland}

Para la conformación del suelo-cemento se utiliza cemento Portland: es una mezcla debidamente proporcionada de materiales arcillosos y calizas, a las que se adicionan yeso natural y agua. Este posee la capacidad de fraguar tanto en aire como en agua una masa endurecida [18]. El cemento Portland debe cumplir con los requisitos físicos establecidos en la NTC 121, los cuales se muestran en la tabla 1.

Tabla 1. Requisitos físicos del cemento Portland

\begin{tabular}{|c|c|c|c|c|c|c|}
\hline & Tipo 1 & Tipo 1M & Tipo 2 & Tipo 3 & Tipo 4 & Tipo 5 \\
\hline $\begin{array}{l}\text { Finura, superficie específica } \\
\text { en } \mathrm{m}^{2} / \mathrm{kg} \text { - ensayo por medio } \\
\text { de permeabilidad al aire, } \\
\text { mínimo }\end{array}$ & 280 & 280 & 280 & - & 280 & 280 \\
\hline $\begin{array}{l}\text { Estabilidad. Expansión en } \\
\text { autoclave, máximo, \% }\end{array}$ & 0,8 & 0,8 & 0,8 & 0,8 & 0,8 & 0,8 \\
\hline \multicolumn{7}{|c|}{ Tiempo de fraguado (Métodos alternativos). } \\
\hline $\begin{array}{l}\text { Ensayo por agujas de Victa: } \\
\text { tiempo inicial, en minutos, } \\
\text { no debe ser inferior a }\end{array}$ & 45 & 45 & 45 & 45 & 45 & 45 \\
\hline $\begin{array}{l}\text { Tiempo final, en horas, no } \\
\text { debe ser mayor que }\end{array}$ & 8 & 8 & 8 & 8 & 8 & 8 \\
\hline
\end{tabular}

Resistencia a la compresión en Mpa (aprox. Kgf/ $\mathrm{cm}^{2}$ )

La resistencia a la compresión de cubos de mortero hechos con una parte de cemento y 2,75 partes de una arena grabada normalizada para este ensayo, preparados y probados de acuerdo con la NTC 220, no debe ser menor que los valores indicados abajo para cada edad

\begin{tabular}{lcccccc}
\hline 1 día & - & - & - & $10,0(100)$ & - & - \\
\hline 3 días & $8,0(80)$ & $12,5(125)$ & $10,5(105)$ & $21,0(210)$ & - & $8,5(85)$ \\
\hline 7 días & $15,0(150)$ & $19,5(195)$ & $17,5(175)$ & - & $7,0(70)$ & $15,5(155)$ \\
\hline 28 días & $24,0(240)$ & - & - & - & $17,5(175)$ & $21,0(210)$ \\
\hline
\end{tabular}

Fuente: Tirano Martínez et al. [19]

Tabla 2. Requisitos químicos del cemento Portland

\begin{tabular}{lcccccc}
\hline & Tipo 1 & Tipo 1M & Tipo 2 & Tipo 3 & Tipo 4a & Tipo 5a \\
\hline Dióxido de silicio (S1O2), mín. \% & - & - & 21,0 & - & - & - \\
\hline Óxido de aluminio (Al2O3), máx. \% & - & - & 6,0 & - & - & - \\
\hline Óxido de hierro (Fe2O3), máx. \% & - & - & 6,0 & - & 6,5 & - \\
\hline Óxido de magnesio (MgO), máx. \% & 7,0 & 7,0 & 7,0 & 7,0 & 7,0 & 7,0 \\
\hline Trióxido de azufre (SO3), máx. \% & 3,5 & 3,5 & - & 4,5 & - & - \\
\hline Pérdida al fuego, máx. \% & - & 5,0 & 4,0 & 4,0 & 3,5 & 4,0 \\
\hline Residuo insoluble, máx. \% & - & 4,0 & 3,0 & 3,0 & 3,0 & 3,0 \\
\hline
\end{tabular}

(continua) 
(viene)

\begin{tabular}{|c|c|c|c|c|c|c|}
\hline & Tipo 1 & Tipo 1M & Tipo 2 & Tipo 3 & Tipo 4a & Tipo 5a \\
\hline $\begin{array}{l}\text { Silicio tricálcico (3CaO. S1O2), } \\
\text { máx. \% }\end{array}$ & - & - & - & - & 35,0 & - \\
\hline $\begin{array}{l}\text { Silicio dicálcico (3CaO. S1O2), } \\
\text { mín. \% }\end{array}$ & - & - & - & - & 40,0 & - \\
\hline $\begin{array}{l}\text { Aluminato tricálcico (3CaO. Al2O3), } \\
\text { máx. \% }\end{array}$ & - & - & 8,0 & 15,0 & 7,0 & 5,0 \\
\hline $\begin{array}{l}\text { (3CaO. S1O2) + (3CaO. Al2O3), } \\
\text { máx. \% }\end{array}$ & - & - & 58,0 & - & - & - \\
\hline \multicolumn{7}{|l|}{$\begin{array}{l}\text { Ferrialuminato tetracálcico más el } \\
\text { doble de aluminato tricálcico }\end{array}$} \\
\hline \multicolumn{7}{|l|}{$\begin{array}{l}\text { (4CaO. Al1O3. Fe2O3) + 2(3CaO. } \\
\text { Al2O3), o solución sólida }\end{array}$} \\
\hline $\begin{array}{l}\text { (4CaO. Al103. Fe2O3) + 2(3CaO. } \\
\text { Al2O3), el que es aplicable, máx. \% }\end{array}$ & - & - & - & - & - & 20,0 \\
\hline
\end{tabular}

Fuente: Tirano Martínez et al. [19]

\subsection{Modificaciones a las mezclas de suelo-cemento}

En las investigaciones revisadas se observó que para realizar la modificación de las propiedades de la mezcla de suelo-cemento se le adicionaron fibras naturales y sintéticas. A continuación se describirán las propiedades de cada una de ellas, junto con sus ventajas y desventajas en la subrasante.

\subsubsection{Subrasante modificada con fibras naturales}

Las fibras naturales o de origen orgánico se pueden obtener a partir de material vegetal o residuos de la industria alimenticia [19]. No obstante, estas son susceptibles de corroerse y degradarse de manera más temprana que las fibras sintéticas, razón por la cual se limita la utilización de este tipo de mezcla a estructuras de un bajo impacto y menor riesgo.

Se ha encontrado que la adición de fibras naturales a la mezcla de suelocemento modifica su rigidez, aumentándola y disminuyendo la deformación. Owokade, Olugbenga, Amu y Opeyemi realizaron ensayos para determinar un método de estabilización del suelo más económico utilizando cáscaras de coco y ceniza del recubrimiento seco de granos o semillas para tres muestras [20]. Donde se obtuvieron valores máximos de densidades en seco (mdd) se incrementaron los valores de fuerza de corte. 
Butt et al. identificaron los efectos en la compactación y el cbr con la adición de fibras de cabello humano. A través de ensayo Proctor determinaron la máxima densidad seca y el contenido óptimo de humedad a diferentes porcentajes de fibra añadida en peso. Con el estudio se determinó que el cbr incrementa para un porcentaje de fibra añadida el peso entre el 0,5 y el $2 \%$ [21].

\subsubsection{Subrasante modificada con adición de fibras sintéticas}

En la ingeniería civil, particularmente en el área de suelos, la técnica de estabilización con polímeros es muy utilizada para mejorar su comportamiento, esfuerzo y deformación [22]. La mayoría de los productos poliméricos promocionados para la estabilización del suelo son acetato de vinilo o copolímeros basados en acrílico; no obstante, una gran cantidad de investigaciones están dirigidas al estudio del cemento, la cal, la mosca ceniza y otros estabilizadores tradicionales [23]. El uso de la cal en la estabilización de suelos está encaminado a mejorar las características naturales del suelo, de modo que aumente su capacidad para resistir los efectos inducidos por el tránsito (esfuerzo de corte) y los cambios volumétricos en diferentes condiciones de clima [24]. Un estudio de condiciones similares a estas últimas es el de Opandalucía, en el que se empleó un polímero en conjunto con cal [25].

La industria de la construcción es un gran consumidor de productos de polímeros. Las previsiones actuales muestran que la demanda de plásticos de los Estados Unidos en los mercados de la construcción crece un 3,6 \% por año, hasta un nivel esperado de 6600 kt en 1995. El pvc es la resina más utilizada debido a su facilidad de procesamiento, costo y ventajas de rendimiento. El poliestireno y los compuestos fenólicos, a su turno, se utilizan en el aislamiento de edificios de espuma de plástico, un mercado que crece a una tasa del $4 \%$ anual. [26]. Un estudio que aplica lo ya mencionado es el de Díaz; en este se evalúa la capacidad de soporte de subrasantes de clase 3 en estado natural y 3 subrasantes a los cuales se les añadió pvc flexible de reciclaje [27].

\section{Resultados}

A partir de la revisión se pudieron identificar los principales objetivos que tienen las investigaciones relacionadas con la adición de polímeros en las mezclas de suelocemento. El enfoque principal de los estudios está orientado a la evaluación de las siguientes propiedades: 
- Resistencia a la compresión

- Resistencia a la tracción

Se estimó que el 65 \% de las investigaciones encontradas dirigen sus estudios a la evaluación de las modificaciones frente a la resistencia a la compresión; y cerca del $30 \%$ se enfocan en estudiar la respuesta a los esfuerzos de tracción cuando se adicionan fibras poliméricas. El 5 \% restante dirige los objetivos a evaluar propiedades como la fatiga o la resistencia a la tensión axial. Casos particulares han buscado entender las modificaciones que la adición de polímeros genera en el suelo-cemento en relación con propiedades como la resistencia a la tensión axial y la reducción de la fatiga del material.

\subsection{Materiales y métodos}

Entre los materiales definidos para los estudios experimentales se encuentran de forma recurrente la mezcla entre el suelo y el cemento. A este respecto, en investigaciones como la de Estabragh y Javadi, el suelo utilizado consistía en un 8 \% de arena, un $55 \%$ de limo y un $37 \%$ de arcilla y el cemento utilizado fue Portland tipo 1 con gravedad específica de $3,15 \mathrm{~g} / \mathrm{cm}^{3}$ [29].

En otros estudios, los suelos han sido más de tipo areno-arcilloso no plástica (sc) de acuerdo con el sistema de clasificación de suelos unificados, tal como el utilizado en [29] y [30]. En este caso, el cemento establecido para la mezcla fue Portland de alta resistencia inicial tipo III, según ASTM C150-07 (2007).

Entre las proporciones de las mezclas se encontraron experimentaciones con $9 \%, 12 \%$ y $15 \%$ de cemento referente al peso de la muestra de suelo [31], siendo estas las más altas encontradas. Otros estudios realizaron pruebas con mezclas de cemento al $5 \%, 7 \%$ y $9 \%$ [20], o de $1 \%, 3 \%$ y $5 \%$ en la investigación de [32]. El porcentaje alude al peso de la muestra de suelo.

Aunque los valores específicos de las características mecánicas, físicas y químicas de estos materiales son ampliamente variables, las especificaciones técnicas generales fueron definidas y la mayoría de los estudios realizan una caracterización de los materiales utilizados en la experimentación, presentando sus resultados de manera similar a como se muestran en la tabla 3. 
Tabla 3. Propiedades de suelo resultado de la caracterización

\begin{tabular}{lc}
\hline \multicolumn{1}{c}{ Property } & Value \\
\hline Liquid limit $(\%)$ & 23 \\
\hline Plasticity index $(\%)$ & 10 \\
\hline Specific gravity & 2,64 \\
\hline Medium sand, according to USCS $(0,425-2,0 \mathrm{~mm})(\%)$ & 6,4 \\
\hline Fine sand, according to USCS $(75-425 \mu \mathrm{m})(\%)$ & 52,2 \\
\hline Fines, according to USCS $(<75 \mu \mathrm{m})(\%)$ & 41,4 \\
\hline Mean particle diameter, $\mathrm{D}_{50}(\mathrm{~mm})$ & 0,12 \\
\hline Coefficient of uniformity & 50 \\
\hline Coefficient of curvature & 0,2 \\
\hline Maximum dry unit weight for modified Proctor & 20,1 \\
\hline Compaction effort $\left(\mathrm{kN} / \mathrm{m}^{3}\right)$ & 10 \\
\hline Optimum moisture content for modified Proctor & \\
\hline Compaction effort $(\%)$ & $\mathrm{SC}$ \\
\hline Uscs class & \\
\hline
\end{tabular}

Fuente: Hurtado [34].

Con respecto a los materiales de refuerzo entre polímeros y resinas, se destacan las adiciones de polisombra abordadas por Tirano Martínez et al. [19] y Moreno Cárdenas et al. [31]. Fibras de polipropileno monofilamento también fueron utilizadas por [29], [34] y [35].

Como elemento innovador se encontró la adición de resinas, las cuales presentan propiedades termoplásticas con buenas propiedades de unión en las investigaciones de [28] y [36].

En general, los porcentajes de polímeros agregados a las mezclas son bajos: se encuentran valores de hasta 0,05\% en peso de la muestra del suelo [31]. En el estudio realizado por Tirado y Moyano [37] se determinó que el diseño óptimo para estabilizar el suelo-cemento con adición de la malla es de 1 \% con relación al peso del suelo seco y un $6 \%$ de cemento.

Con respecto a los métodos de evaluación utilizados se pudo observar que son variados, según el lugar de origen de la investigación. Esto debido a que diversos países tienen estas metodologías estandarizadas, por lo que se adoptan para las investigaciones.

Entre las pruebas y análisis desarrollados se encuentran los siguientes:

- $\quad$ Análisis granulométrico de suelos por tamizado (I. N. V. E - 123)

- Determinación del límite líquido de los suelos (I. N. V. E - 125) 
- Límite plástico e índice de plasticidad (I. N. V. E - 126)

- Determinación de los factores de contracción de los suelos (I. N. V. E - 127)

- Determinación del peso específico y de la llenante mineral. (I.N.V. E - 128)

- Relaciones de peso unitario - humedad en los suelos (I.N.V. E - 142)

- Relación de soporte del suelo en el laboratorio (cbr de laboratorio), I. N. V. E - 148

- Equivalente de arena (I. N. V. E - 133)

- Desgaste en la máquina de los ángeles (I. N. V. E - 218)

- Sanidad de los agregados frente a la acción de las soluciones de sulfato de sodio o de magnesio (I. N. V. E - 220)

- Prueba de presión no confinada, de acuerdo con ASTM D 1633.

- $\quad$ Pruebas de tensión de separación según el estándar brasileño NBR 7222

- Estándar brasileño NBR 5739

\section{Discusión de resultados}

La recopilación de los resultados se presenta clasificada en dos ítems, según la propiedad evaluada en el momento de la adición de los polímeros y la naturaleza de la fibra añadida.

\subsection{Resistencia a la compresión}

En las investigaciones se ha encontrado que la relación entre la resistencia a la compresión y el porcentaje de adición de elementos en la mezcla no es directa (relación de resistencia vs. porcentaje de cemento). Se considera que la resistencia no aumenta con respecto a dicha dosificación [31]. Los resultados arrojaron una mayor resistencia con una adición del 9 \% de cemento; sin embargo, se recomienda analizar más puntos con el fin de identificar el comportamiento característico de esta propiedad. Al añadir la polisombra a la mezcla, se determinó que el comportamiento de la resistencia es de tipo parabólico: se obtuvo el punto óptimo con la inclusión del 1 \% de polisombra. Pese a lo anterior, Moreno Cárdenas y Linares reemplazaron una parte de material por las fibras de polisombra (0,05\%), con lo cual demostraron que, a mayor cantidad de cemento agregado a la mezcla, la resistencia del suelo aumenta [31].

En relación con la adición de fibras, se estableció que esta incrementa de manera moderada el punto de fuerza máxima del suelo.

En el estudio de Estabragh et al. se evidencian variaciones significativas en el comportamiento: se estableció que el efecto de 0,5\%, 0,75\%, 1,0 \% y 1,25\% de fibra 
incrementa la resistencia en $8 \%, 15,7 \%, 18,5 \%$ y $21 \%$, respectivamente [38]. Otros estudios muestran que variables adicionales, como la longitud de las fibras adicionadas a las mezclas de suelo-cemento, también tienen un efecto importante en la modificación de la resistencia a la compresión [29].

Respecto a las variaciones de la fuerza máxima contra la longitud de la fibra para el suelo-cemento con $10 \%$ inclusión de cemento y fibra de 0,5\% y 1 \% en diferentes tiempos de curado, se observan las variaciones de la fuerza máxima contra la longitud de la fibra para un suelo con $8 \%$ de adición de cemento. Como se evidencia en Estabragh et al., el ensayo con mayor resistencia a la fuerza aplicada es el suelo con adición de $1 \%$ de fibra y 28 días de curado. Esto significa que al incrementarse el tiempo de curado, la resistencia del material aumentará; y lo mismo ocurre con la adición de porcentaje de fibra y su longitud de esta, dado que a longitudes inferiores y superiores a $10 \mathrm{~mm}$, la resistencia disminuye [39].

Estabragh et al. [39] demostraron que la adición de resina en un $10 \%$ a las mezclas de suelo-cemento incrementa la resistencia a la compresión. Describen, además, las variaciones que tiene esta propiedad con respecto a la adición de resina y al tiempo de curado. Este último es otra de las variables con presencia reiterada en los estudios: se ha encontrado que influye en los valores monitoreados, aunque no ha sido el principal interés de investigación. Los resultados del estudio de Torres arrojan valores similares [40].

La mejor resistencia a la compresión se produce con 75 \% de pet. La mezcla experimental logra obtener la resistencia entre 3,5 y $7 \mathrm{Mpa}$, haciendo viable su uso en las bases estabilizadas con cemento y en cualquier porcentaje de sustitución en pet. En conclusión, la mezcla con mejor comportamiento es de 75 \%, ya que, en primera instancia, el artículo 351 de Invías exige un cumplimiento con la resistencia a la comprensión y el 75 \% en PET cumple con la resistencia mínima de 3,5 MPa [41]; Meneses y Fuentes, Alesmar Luis et al. y Khan presentaron estudios relacionados con esto [42], [43], [44].

Mirzababaei, Arulrajahb y Ouston utilizaron dos aditivos químicos, poli (alcohol vinílico, es decir, pva) y ácido 1,2,3,4-butanotetracarboxílico (btca) en suelos arcillosos con un estado relativamente rígido (es decir, un peso de unidad seca de 16,2 kN / m³) y en estado relativamente suave (es decir, con peso unitario seco de 10,8 kN / m³) [45]. El contenido óptimo de PVA para la estabilización del suelo dependía del peso unitario seco, la relación de vacío inicial y el contenido de agua de la muestra de suelo. La adición de 1 \% de PVA condujo a un aumento moderado en el ucs de muestra de suelo con peso unitario seco de 16,2 kN / m3 (es decir, e0: 0,64, $\omega=16,8 \%$ ). 
Para muestras con un peso unitario seco de 10,8 kN / m³ (es decir, e0: 1,46, $\omega=48 \%$ ), el ucs aumentó con el contenido de PVA al 1,5\%. [45] investigó sobre el efecto de la adición de pva y btca (1,2,3,4 ácido butacarboxílico) en un suelo arcilloso expansivo. Se observó el efecto de estos polímeros sobre la resistencia y la compresión no confinada en muestras de suelo preparadas con peso seco máximo (16,2 kN / m3 y 17\% de contenido de agua) y peso unitario seco menor (10,8 kN / m³ y $48 \%$ de contenido de agua), agregados en dosis de 0,1 \% a 1,5\% y 0,1 \% a 0,5\% y curados de 1 a 14 días.

Además de usarse las nombradas fibras sintéticas, en años recientes se han desarrollado investigaciones en las que materiales de origen natural se han usado para sustituir y reforzar las mezclas de subrasante. Yadav y Tiwari utilizaron diversos porcentajes de cenizas de estanque de arcilla, las cuales se agregaron a la mezcla del cemento Portland previamente estabilizado y suelo arcilloso proveniente de la zona de estudio; cabe resaltar que las mezclas elaboradas fueron curadas por un periodo de 28 días [46].

Respecto a las variaciones de estrés -arcilla mezclada (a) con ceniza de estanque solamente (b) con ceniza de estanque y estabilizada con $2 \%$ y $4 \%$ de cemento (después de 28 días de curado), * PA- ceniza de estanque, C-cemento, se graficaron los valores de presión ejercidos a los diferentes ensayos realizados con ceniza de estanque. Los porcentajes con mayor resistencia a la compresión fueron 10 \% y $20 \%$ de sustitución; es decir, los picos de estrés que se generaron son muy similares al ensayo con $0 \%$ de ceniza. Por tal razón, son porcentajes óptimos para la sustitución de la arcilla con ceniza, los cuales no aportarán cambios significativos a la resistencia de la subrasante [46]. Por otro lado, el ensayo en el que se adicionó 30 \% de ceniza tuvo una caída en su pico de estrés en comparación con los demás; es decir, de 121,7 kPa del ensayo con el 20 \% de adición, el punto máximo fue de 102,61 kPa, a lo que se le atribuye que las partículas subredondeadas de la ceniza junto a las partículas irregulares del suelo generan un deslizamiento entre ellas. Esto indica que no es un porcentaje adecuado para la sustitución de la arcilla, dado que hace más frágil al material.

Los resultados de la evaluación de la resistencia de los ensayos realizados con mezcla de ceniza y cemento indican que los ensayos con porcentajes de $10 \%$ y $20 \%$ de ceniza de estanque con adición de $4 \%$ de cemento son aquellos que tuvieron mayor resistencia a la presión ejercida. Cabe resaltar que estas mezclas tuvieron mayor resistencia en comparación con aquellas que no tenían cemento, con lo cual se tiene que las mezclas cementadas ostentan mejor resistencia y son las más apropiadas para la elaboración de subrasantes. 
Por otro lado, Paul y Sneha estudiaron el comportamiento de la compactación y la resistencia de un suelo tratado con cenizas volantes junto con fibras de bambú [47]. En la curva de deflexión de carga de suelo mezclado con diferentes porcentajes de ceniza se observa que la mezcla óptima para la sustitución del suelo es de 20 \% de ceniza, dado que fue la mezcla que resiste más la presión ejercida.

En cuanto a la curva de desviación de un suelo mezclado con el $20 \%$ de cenizas volantes y diferentes porcentajes de fibra de bambú, hay un aumento significativo en la resistencia a la compresión debido a la inclusión de fibra de bambú en el suelo tratado con porcentaje óptimo de cenizas volantes (20\%). El aumento en la fuerza se muestra en la curva de desviación de un suelo mezclado con el 20 \% de cenizas volantes y diferentes porcentajes de fibra de bambú . Así entonces, los porcentajes óptimos de la mezcla son un 20 \% de cenizas volantes junto con 1 \% de fibra de bambú.

\subsection{Resistencia a la tracción}

Otra de las propiedades que ha sido estudiada es la resistencia a la tracción. Según Consoli, Moraes y Festugato, la resistencia a la tracción dividida (qt en las gráficas) aumentó linealmente con la cantidad de cemento (C), mientras que disminuyó con el aumento de la porosidad de las mezclas compactadas en la relación de la resistencia a la tracción de la mezcla vs. el porcentaje de cemento (a) [33]. A partir de la medición de esta propiedad sobre mezclas de suelo-cemento modificadas con diferentes proporciones de fibras se generaron gráficas como las de la relación de la resistencia a la tracción de la mezcla vs. porcentaje de cemento (b), en las que se evidencia el aumento en la resistencia a la tracción dividida del suelo cementado para toda la gama de cemento estudiada [33]. A pesar de que las diferencias encontradas son claras, los investigadores presentaron la variación de resistencia a la tracción dividida con ajuste relación vacío/cemento para muestras no reforzadas y especímenes reforzados con fibra, en las que se evalúa una curva de comportamiento de suelo-cemento con y sin la adición de fibras. En dicha gráfica resulta claro el aumento de la resistencia a la tracción en suelos cementados por la adición de fibras poliméricas.

Otro estudio que comparte un análisis similar es el realizado por Torres; en este se buscó el mejoramiento de las mezclas asfálticas para pavimentación por medio del uso de residuos de Hule como llantas, SBR, hule natural, polibutadieno y hule reciclado [48].

En el 2017, Festugato et al. lograron identificar que la adición de fibras lograba un aumento de hasta el 67 \% en la resistencia a la tracción de mezcla de suelo-cemento estudiada. La relación encontrada también estaba relacionada de manera directa con 
la longitud de las fibras adicionadas: se encontró dicho porcentaje máximo de incremento en la resistencia con la adición de fibras entre 0 mm y 24 mm [29].

Finalmente, Anagnostopoulos logró identificar a través de la adición de resina epóxica que después de 90 días de curado se lograba un incremento de hasta el 20\% para la resistencia a la tracción de la mezcla de suelo-cemento estudiada [36].

En el estudio realizado por Leiva, se utilizaron bolsas de polietileno fundido como agente estabilizador, y el porcentaje óptimo fue $6 \%$ con respecto al peso seco del suelo [49]. El cbr del suelo arcilloso es 4,145\% al $95 \%$ de la máxima densidad seca; y con la adición de bolsas de polietileno fundido en forma de grumos en una dosificación de $6 \%$ del peso seco del suelo, se incrementó el cbr a 7,98\%, al $95 \%$ de la máxima densidad seca.

Zhang, Cai y Liu basaron sus experimentos en la aplicación de lignina como estabilizante en una subrasante de una avenida principal en Yancheng (República Popular China). El estudio se basó en el análisis de tres secciones de la vía, denominadas $A$, B y $C$, con $12 \%$ y $8 \%$ de lignina para las secciones A y B, respectivamente, y $8 \%$ de limo para la sección $C$. Los resultados permitieron establecer que, pasados 8 días de curado de la mezcla, la sección A incrementó su cbr de 50,6 \% a 87,7 \%; y la sección $B$, de 40,3 \% a 70,6 \% [50].

\subsection{Reducción de la fatiga}

Lenoir llevó a cabo una investigación con la cual determinó las variaciones en la fatiga de mezclas de suelo-cemento por la adición de polímeros [30]. Los estudios que se realizaron se desarrollaron con 2 tipos de suelos: de un lado, arcilla arenosa de grano fino; y de otro, un suelo con material de grano grueso y un pequeño contenido de arcilla. Se utilizaron fibras de cáñamo con un diámetro de 10 a 20 mm, con propiedades mecánicas de resistencia a la tracción de 310 y 750 MPa. Con este trabajo nombrado fue posible determinar que la resistencia a la flexión aumenta ligeramente con la adición de fibras; los estudios realizados alcanzaron 0,805 MPa con una desviación estándar (std) de 0,127 MPa, lo que que representa una ganancia del $16 \%$ con 0,2 \% de fibras. De la investigación se deduce que la adición de 0,3 \% de fibras mejora los rendimientos mecánicos de fatiga del suelo estabilizado, al tiempo que mejora la homogeneidad del material.

\subsection{Resistencia a la tensión axial}

Con el fin de identificar los cambios inducidos en las diferentes muestras de suelocemento referentes a la tensión axial, se realizaron tres grupos de pruebas sobre 
muestras reforzadas con cuatro contenidos de fibra diferentes $(0,5 \%, 0,75 \%, 1 \%$ y 1,25\%); muestras estabilizadas con cemento con tres contenidos diferentes de cemento ( $5 \%, 8 \%$ y $10 \%$ ); y muestras estabilizadas con cemento y reforzadas con fibra, con los mismos contenidos de fibra que las reforzadas sin cementar.

Para Hinojosa y Paú, la capacidad portante en términos de cbr para un suelo de baja capacidad portante con presencia de polímeros reciclados pet aumenta $26 \%$ en promedio, mientras que la expansión disminuye en promedio en 1,5 \% [51]. La carencia de gravas en los suelos estudiados hace que el suelo tenga poca resistencia al corte, como se pudo comprobar con el ensayo de cbr, razón por la cual al adicionar elementos resistentes como pet hace que el suelo tenga mayor fricción y, por ende, presente mayor resistencia al corte.

Estabragh, Namdar y Javadi, lograron identificar que el efecto de 0,5 \%, 0,75 \%, $1,0 \%$ y $1,25 \%$ de fibra sobre el aumento de la resistencia máxima es del $8 \%, 15,7 \%$, $18,5 \%$ y $21 \%$, respectivamente [38]. Los suelos reforzados alcanzaron el pico de estrés en deformaciones axiales entre $2 \%$ y $4 \%$.

\section{Conclusiones}

Se denota un aumento considerable en la resistencia de compresión, que varía según el tipo o material a mezclar con el suelo. En términos de eficiencia se requiere un $9 \%$ de cemento, y respecto de la adición de fibras se requiere $10 \%$ de cemento con adición de 0,75 \% de fibra variando, si se desea un aumento hasta en un $35 \%$. Asimismo, se requieren fibras con una longitud de $24 \mathrm{~mm}$. En cuanto a la utilización de resina, se requiere de un $10 \%$ a la mezcla de suelo-cemento. Vargas Quispe ha podido obtener resultados con fibras de $40 \mathrm{~mm}$ y una dosificación de 0,50 \% y 0,75\%, con incrementos del cbr de hasta $28 \%$ del valor inicial [52]. Todos estos porcentajes están relacionados con el peso de la muestra de suelo. Estudios similares, como los de Hinojosa y Rajesh, arrojan valores similares [53], [54].

Modelos analíticos y físicos como los desarrollados por Love et al. muestran que el reforzamiento producido por las geomallas tiende a reducir los esfuerzos de corte transmitidos a la superficie de la subrasante. Cuando este reforzamiento no está presente, estos esfuerzos son transmitidos de manera directa a la subrasante, reduciendo su capacidad a las cargas verticales [55].

En cuanto a fibras naturales se observa que, conforme se adiciona cemento a la mezcla, la resistencia de este mejora. El bambú y las cenizas aportan mayor resistencia a la subrasante. De otro lado, debido a las características hidrofílicas de 
los suelos arcillosos expansivos, se denota mejora la resistencia a la compresión. Sin embargo, la eficacia depende, en su mayoría, del peso unitario del suelo.

La resistencia a la tracción presenta un aumento lineal con la cantidad de cemento; caso contrario a lo que ocurre en presencia del aumento de la porosidad. Con la adición de fibras se logra un aumento de hasta el 67 \% con fibras de longitud de $24 \mathrm{~mm}$. Tras 90 días de curado, la resina epóxica logra un incremento de hasta el $20 \%$. Para suelos con arcilla arenosa de grano fino y con material de grano grueso, se puede tener un aumento de la resistencia a la flexión de 0,688 MPa a 0,805 MPa del suelo-cemento en relación con el suelo cementado adicionado con fibras, con un $0,3 \%$ de fibras en porcentaje al volumen del suelo. Para las muestras reforzadas con cuatro contenidos de fibra diferentes $(0,5 \%, 0,75 \%, 1 \%$ y $1,25 \%)$ se identificaron aumentos de la resistencia máxima de $8 \%, 15,7 \%, 18,5 \%$ y $21 \%$, respectivamente; estos suelos logran alcanzar un pico de estrés en deformaciones axiales de $2 \%$ y $4 \%$.

A partir de la revisión realizada se puede afirmar que la inclusión de polímeros en los materiales de subrasante genera una mejora en las propiedades evaluadas. Las investigaciones encontradas, tanto del ámbito nacional como del internacional, revelan que los materiales poliméricos evaluados son muy diversos y, por lo tanto, se considera necesario profundizar en el estudio sobre la aplicación de cada uno de ellos. A este respecto, la recopilación de información presentada es de gran importancia para la comunidad académica, puesto que genera puntos de partida actualizados para próximas investigaciones en el campo. De manera complementaria, se considera fundamental ampliar las investigaciones hacia la adición de polímeros derivados del reciclaje de materiales, con el fin de contribuir a la mejora de la huella ecológica en el campo de la construcción -y de modo específico, de vías en el país-.

\section{Referencias}

[1] C. J. Toirac, "El suelo cemento como un material de construcción”, Ciencia y sociedad, vol. xxxiii, n. 4 , pp. 41-63, 2008. doi: http://dx.doi.org/10.22206/cys.2008.v33i4.

[2] M. P. Gatani, "Ladrillos de suelo-cemento: Mampuesto tradicional en base a un material sostenible”, Informes de la construcción, n. 51, pp. 35-47, 2000 [En línea]. Disponible en: https://doaj.org/article/bf58955688a040feae79f1bf9b237573.

[3] P.P. Raj, “Ground improvementtechniques”, National Conference on Advances in Geotechnical Engineering, pp. 35-42, 2005. doi: https://10.13140/RG.2.1.4865.4965. 
[4] Y. Laurente Ronceros, Estudio comparativo del mejoramiento de la subrasante y base de la carretera Cañete-Chupaca tramo Km 220+000 - Km 240-000. Lima: Universidad Nacional de Ingeniería, Lima, 2011. Recuperado: http://cybertesis.uni.edu.pe/handle/uni/3483.

[5] J. B. Hernández-Zaragoza, J. Horta Rangel, V. Castaño, A. Coronado y A. Teresa López-Lara, "Polímeros para la estabilización volumétrica de arcillas expansivas" Revista Iberoamericana de Polímeros, vol. 11, n. ³, pp. 159-168, 2010 [En línea]. Disponible en: http://dialnet.unirioja. es/servlet/oaiart?codigo=3694127.

[6] M. Sol-Sánchez. M. C. Rubio-Gámez y F. Moreno-Navarro, "Structural analysis of polymer modified bituminous materials in the rehabilitation of light-medium traffic volume roads", Construction and Building Materials, n. ${ }^{\circ}$ 156, pp. 621-631, 2017. doi: https://doi.org/10.1016/j. conbuildmat.2017.09.006.

[7] G. Ayala y C. Terreros, Estabilización y control de suelos expansivos utilizando polímeros. Samborondon, Ecuador: Universidad de Especialidades Espíritu Santo (uees), pp. 1-115, 2017 [En línea]. Disponible en: http://repositorio.uees.edu.ec/123456789/1945

[8] A. Hedge, "Geocell reinforced foundation beds-past findings, present trends and future prospects: A state-of-the-art-review", Construction and Building Materials, vol. 154, pp. 658-674, 2017. doi: https://doi.org/10.1016/j.conbuildmat.2017.07.230

[9] V. Sierra y M. Duarte, Estudio del comportamiento de un material de subrasante típico de Bogotá estabilizado con un sistema de geoceldas ante la aplicación de ciclos de carga y descarga mediante pruebas de laboratorio. Bogotá: Pontificia Universidad Javeriana, pp. 8-54, 2011. Disponible en: http://hdl.handle.net/10554/7505.

[10] E. V. Cuelho y E. S. Perkins, "Geosynthetic subgrade stabilization - Field testing and design method calibration", Transportation Geotechnics, n. ${ }^{\circ}$ 10, pp. 22-34, 2017. doi: https://doi.org/10.1016/j.trgeo.2016.10.002.

[11] C. Geiman, Stabilization of soft clay subgrades in Virginia phase I Laboratory study. Blacksburg, Virginia Polytechnic Institute and State University, 2005 [En línea]. Disponible en: https://vtechworks.lib.vt.edu/handle/10919/32499.

[12] F. H. Khan, "Analysis of the influence of waste polymer on soil subgrade" International Research Journal of Engineering and Technology, n. 3, pp. 1810-1817, 2016 [En línea]. Disponible en: https://www.irjet.net/archives/V3/i3/IRJET-V313379.pdf. 


\section{Erika Julieth Serrano Rodríguez, Edgar Alexander Padilla González}

[13] D. F. Lin y Li Chen, "Stabilization Treatment of Soft Subgrade Soil by Sewage Sludge Ash and Cement”, Journal of Harzardous Materials, vol. 162, n. ${ }^{1}$, pp. 321-327, 2008. doi: https://doi. org/10.1016/j.jhazmat.2008.05.060.

[14] R. Hufenus, R. Rueegger, R. Banjac, P. Mayor, S.Springmany R. Bronnimann, “Full-scalefield tests on geosynthetic reinforced unpaved roads on soft subgrade", Geotextiles and Geomembranes, vol. 24, pp. 21-37, 2006. doi: https://doi.org/10.1016/j.geotexmem.2005.06.002.

[15] V. T. N. Perugachi, Las características del suelo de subrasante de los caminos vecinales de la comunidad de Echaleche Pilahuín y su incidencia en el comportamiento de la capa de rodadura. Ambato, Ecuador: Universidad Técnica de Ambato, pp. 1-120, 2011 [En línea]. Disponible en: http://repositorio.uta.edu.ec/handle/123456789/2200.

[16] Instituto Nacional deVías-Invías, “Artículo341-07”,2007 [En línea]. Disponibleen:ftp://ftp.unicauca.edu.co/Facultades/FIC/IngCivil/Especificaciones_Normas_INV-07/Especificaciones/ Articulo341-07.pdf.

[17] C. Aguilar, Cemento Portland: fabricación, propiedades y empleo. México: Instituto Mexicano del Cemento y del Concreto, pp. 12-25, 1970. Recuperado: http://imcyc.com/redcyc/imcyc/ biblioteca_digital/tecnologia_del_concreto.pdf

[18] Instituto Colombiano de Normas Técnicas - Icontec, NTC-121. Bogotá: Icontec, pp. 2-24, 2005.

[19] A. D. Tirano Martínez, C. D. Moyano Cobos y M. E. Riascos Caipe, "Análisis de la resistencia y durabilidad de un suelo-cemento adicionando material no biodegradable polietileno malla Raschel (polisombra) reciclada en diferentes porcentajes en relación con el peso del suelo", tesis de grado, Universidad de la Salle, Bogotá, 2016, pp. 15-95 [En línea]. Disponible en: http://hdl.handle.net/10185/20564

[20] O. Amu, O. S. Owokade y O. Shitan, "Potentials of Coconut Shell and Husk Ash on the Geotechnical Properties of Lateritic Soil for Road Works", International Journal of Engineering and Technology, vol. 3, n. ${ }^{\circ}$ 2, pp. 87-94, 2011 [En línea]. Disponible en: https://www.idc-online.com/technical_references/pdfs/civil_engineering/Potentials\%20of\%20Coconut.pdf.

[21] W. Butt, K. Gupta, H. Naik y S. Bhat, "Soil Sub- grade Improvement Using Human Hair Fiber", International Journal of Scientific \& Engineering Research, vol. 5, pp. 977-981, 2014 [En línea]. Disponible en: https://www.ijser.org/researchpaper/Soil-Sub-grade-Improvement-UsingHuman-Hair-Fiber.pdf. 
[22] J. Díaz y J. Mejía, "Estabilización de suelos mediante el uso de un aditivo químico a base de compuestos inorgánicos", trabajo de grado, Facultad de Ingenierías Fisicomecánicas, Universidad Industrial de Santander, Bucaramanga, pp. 5-63, 2004 [En línea]. Disponible en: http://docplayer.es/11977568-Estabilizacion-de-suelos-mediante-el-uso-de-un-aditivo-quimico-a-base-de-compuestos-inorganicos.html

[23] K. Newman y J. S. Tingle, "Emulsion polymers for soil stabilization", en WorldwideAirportTechnologyTransferConference,AtlanticCity,pp.5-65,2004[Enlínea]. Recuperado: http://www.airporttech.tc.faa.gov/Airport-R-D/Conference-and-Workshop/Airport-RDConference-Detail/dt/Detail/ItemID/429/Emulsion-Polymers-for-Soil-Stabilization

[24] J. R. Menéndez Acurio, Ingeniería de Pavimentos. Materiales, Diseño y Conservación. Lima, Perú: Departamento de Imprenta de ICG, 2012, pp. 15-64.

[25] Agencia de Obra Pública de la Junta de Andalucía, "Utilización de polímeros en la estabilización de suelos para su uso en carreteras, ejecución de un tramo experimental en el enlace de jédula de la A-382", 2007, pp. 1-18 [En línea]. Disponible en: http://www.opandalucia.es/ giasa_datos/documentos_bd/Documento\%206.pdf.

[26] C. J. Kibert, "Construction materials from recycled polymers", Proceedings of the Institution of Civil Engineers - Structures and Buildings, vol. 99, n. ${ }^{\circ} 4$, pp. 455-464, 1993 [En línea]. Disponible en: http://www.icevirtuallibrary.com/content/serial/stbu

[27] E. R. M. Díaz, Evaluación de la capacidad de soporte de subbases granulares tipo 3, con la adición de PVC reciclado a base de residuos de ropa industrial. Quito, Ecuador: Pontificia universidad Católica del Ecuador, 2016, pp. 5-54 [En línea]. Disponible en: http://repositorio. puce.edu.ec/handle/22000/11249.

[28] A. Estabragh, M. Naseh, I. Beytolahpour y A. Javadi, "Strength of a clay soil and soil-cement mixture with resin”, Ground Improvement, vol. 166, n. ${ }^{\circ}$ GI2, pp. 108-113, 2012 [En línea]. Disponible en: https://ore.exeter.ac.uk/repository/bitstream/handle/10871/21355/ Strength\%20of\%20a\%20clay\%20soil\%20and\%20soil-cement\%20mixture\%20with\%20resin.pdf?sequence=1.

[29] L. Festugato, E. Menger, F. Benezra y E. A. Kipper, "Fibre-reinforced cemented soils compressive and tensile strength assessment as a function of filament length", Geotextiles and Geomembranes, vol. 45, n. ${ }^{\circ}$ 1, pp. 77-82, 2017. doi: https://doi.org/10.1016/j. geotexmem.2016.09.001. 
[30] T. Lenoir, M. Preteseilley S. Ricordel, "Contribution of the Fiber Reinforcement on the Fatigue Behavior of Two Cement-modified Soils”, International Journal of Fatigue, vol. 93, n. ${ }^{\circ}$ 1, pp. 71-81, 2016. doi: https://10.1016/j.ijfatigue.2016.08.007.

[31] B. G. Moreno Cárdenas, O. Ibarra Linares y M. E. Riascos Caipe, "Ensayo Proctor estándary modificado para mezcla suelo cemento con adición de polisombra", tesis de grado, Universidad de la Salle, Bogotá, pp. 5-26, 2016 [En línea]. Disponible en: http://repository.lasalle.edu.co/ bitstream/handle/10185/22391/40121091_2017_P1.pdf?sequence=1\&isAllowed=y.

[32] E. Rodríguez Rincón, H. A. Rondón Quintana, D. M. Vélez Pinzón y L. C. Aguirre, "Influencia de la inclusión de desecho de PVC sobre el CBR de un material granular tipo subbase", tesis de grado, Facultad de Ingenierías, Universidad de Medellín, Medellín, pp. 3-24, 2006 [En línea]. Disponible en: https://revistas.udem.edu.co/index.php/ingenierias/article/view/232.

[33] N. C. Consoli, R. R. Moraes y L. Festugato, "Split tensile strength of monofilament polypropylene fiber-reinforced cemented sandy soils”, Geosynthetics International, vol. 18, n. ${ }^{\circ}$ 2, pp. 57-62, 2011. doi: https://doi.org10.1680/gein.2011.18.2.57.

[34] D. R. Hurtado, "Mejoramiento de suelos expansivos mediante inclusiones de fibras", tesis de grado, Universidad Autónoma de Querétaro, Querétaro, pp. 1-69, 2014 [En línea]. Disponible en: http://hdl.handle.net/123456789/1811.

[35] B. Kalantari y B. Huat, "Peat soil stabilization, using Ordinary Portland Cement, Polypropylene fibers, and Air Curing Technique", The European Journal of Government and Economics, vol. 13, pp. 1-13, 2008 [En línea]. Disponible en: https://www.researchgate. net/publication/242258317_Peat_soil_stabilization_using_Ordinary_Portland_Cement_ Polypropylene_fibers_and_Air_Curing_Technique.

[36] C. A. Anagnostopoulos, "Strength properties of epoxy resin-soil-cement mixtures", Construction Materials, vol. 170, n. ${ }^{\circ}$ 3, pp. 123-133, 2016. doi: https://10.1680/jcoma.14.00023.

[37] A. Tirado y C. Moyano, "Análisis de la resistencia y durabilidad de un suelo-cemento adicionando material no biodegradable polietileno malla Raschel (polisombra) reciclada en diferentes porcentajes en relación con el peso del suelo", tesis de grado, Universidad de la Salle, Bogotá, 2016 [En línea]. Disponible en: http://hdl.handle.net/10185/20564.

[38] R. Estabragh, P. Namdar y A. Javadi, "Behavior of cement-stabilized clay reinforced with nylon fiber" Geosynthetics International, vol. 19, n. ${ }^{\circ}$ 1, pp. 85-92, 2012. doi: https://10.1680/ gein.2012.19.1.85. 
[39] A. R. Estabragh, S. Ranjbari y A. Javadi, "Properties of a Clay Soil and Soil-Cement Reinforced with Polypropylene Fibers", Aci Materials Journal, vol. 2, n. ${ }^{\circ}$ 114, pp. 2-41, 2017. doi: https:// doi.org/10.14359/51689469.

[40] J. Galindo, "Evaluar la inclusión de micropolímeros y cemento Portland como agente estabilizador de suelos para conformación de rasantes", tesis de grado, Universidad Militar Nueva Granada, Bogotá, pp. 1-16, 2018 [En línea]. Disponible en: http://hdl.handle.net/10654/17095.

[41] D. Bustos y J. Sarmiento, "Análisis del comportamiento de base granular estabilizada con cemento sustituyendo con material no biodegradable tereftalato de polietileno (pet) en la fracción del agregado que pasa el tamiz \# 4 y se retiene en el \# 16”, trabajo de grado, Universidad de la Salle, Bogotá, pp. 4-89, 2016 [En línea]. Disponible en: http://hdl.handle. net/10185/18915.

[42] L. Meneses y L. Fuentes, "Base estabilizada con cemento modificada con pet reciclado", trabajo de grado, Universidad de la Salle, Bogotá, pp. 2-57, 2015. Disponible en: http://hdl.handle.net/10185/17405.

[43] A. Luis, N. Rendón y M. Korody, "Diseños de mezcla de tereftalato de polietileno (pet) - cemento", Revista de la Facultad de Ingeniería, Universidad Central de Venezuela, vol. 23, n. ${ }^{1}$, pp. 77-86, 2008 [En línea]. Disponible en: http://saber.ucv.ve/ojs/index.php/rev_fiucv/article/ view/5048/4855.

[44] N. Prakhash, D. Kumar, N. Guruvignesh, A. Harichandra y D. Muthukumar, "Soil Stabilization by Using Waste Plastic". International Journal of Advance Research in Science and Engineering, vol. 7, n. ${ }^{\circ}$ 4, pp. 317-326, 2016 [En línea]. Disponible en: http://www.ijarse.com/images/fullpdf/1524229680_IEl1034ijarse.pdf.

[45] M. Mirzababaei, A. Arulrajah y M. Ouston, "Polymers for Stabilization of Soft Clay Soils", Procedia Engineering, n. ${ }^{\circ}$ 189, pp. 25-32, 2017. doi: https://10.1016/j.proeng.2017.05.005.

[46] J. S. Yadav y S. K. Tiwari, "Behaviour of cement stabilized treated coir fibre-reinforced claypond ash mixtures", Journal of Building Engineering, vol. 8, pp. 131-140, 2016. doi: http://10.1016/j.jobe.2016.10.006.

[47] J. Paul y A. R. Sneha, "Effect of random inclusion of bamboo fibers on strength behavior of flyash treated black cotton soil”, International Journal of Civil Engineering and Technology, vol. 7, n 5, pp. 153-160, 2016 [En línea]. Disponible en: http://www.iaeme.com/MasterAdmin/ uploadfolder/IJCIET_07_05_017/IJCIET_07_05_017.pdf. 
[48] L. R. V. Torres, Diseño de pavimentos flexibles con superficie de rodamiento conformada con asfaltos ahulados y reforzados con polímeros. México: Escuela Superior de Ingeniería y Arquitectura, 2011 [En línea]. Disponible en: http://tesis.ipn.mx:8080/xmlui/ handle/123456789/9669.

[49] R. Leiva, "Utilización de bolsas de polietileno para el mejoramiento de suelo a nivel de la subrasante en el Jr. Arequipa, progresiva km 0+000 - km 0+100”, tesis de grado, Universidad Nacional del Centro del Perú, Distrito de Orcotuna, Concepción, Huancayo, 2016. Recuperado: http://repositorio.uncp.edu.pe/handle/UNCP/1181.

[50] T. Zhang, G. Cai y S. Liu, "Application of lignin-based by-product stabilized silty soil in highway subgrade: A field investigation”, Journal of Cleaner Production, vol. 142, pp. 4243-4257, 2017. Disponible en: https://doi.org/10.1016/j.jclepro.2016.12.002.

[51] R. Hinojosa y G. Paúl, Mejoramiento de subrasantes de baja capacidad portante mediante el uso de polímeros reciclados en carreteras, tesis de grado, Universidad Nacional del Centro del Perú, Distrito de Orcotuna, Concepción, Huancayo, pp. 1-103, 2014 [En línea]. Disponible en: http://repositorio.uncp.edu.pe/handle/UNCP/416.

[52] N. Vargas, "Efecto de la adición de fibra de polímeros reciclados en el valor de cbr de suelos granulares en pavimentos”, tesis de grado, Escuela Académica Profesional de Ingeniería Civil, Huancayo, pp. 5-102, 2017 [En línea]. Disponible en: http://repositorio.continental.edu.pe/ handle/continental/3371.

[53] G. P. Ramos Hinojosa, "Mejoramiento de subrasantes de baja capacidad portante mediante el uso de polímeros reciclados en carreteras", tesis de grado, Universidad Nacional del Centro del Perú, Huancayo, pp. 1-103, 2014. [En línea]. Disponible en: http://repositorio.uncp.edu. pe/handle/UNCP/416.

[54] U. Rajesh, S. Sajjaab y V. Chakravarthi, "Studies on Engineering Performance of Geogrid Reinforced”, Transportation Research Procedia, vol. 17, pp. 164-173, 2016. doi: https://10.1016/j.trpro.2016.11.072

[55] J. Love, H. Burd, W. Milligan y G. Houlsby, "Analytical and model studies of reinforcement of a layer of granular fill on a soft clay subgrade", Geotech, vol. 24, pp. 611-622, 1987 [En línea]. Disponible en: http://www.nrcresearchpress.com/doi/abs/10.1139/t87-075\#. w9zwepMzbDc. 